

\section{University of the Highlands and} Islands

BSc Oral Health Science

https://www.uhi.ac.uk/en/courses/bsc-oralhealth-science/

MSc Oral Health Science

https://www.uhi.ac.uk/en/courses/msc-oralhealth-science/

PG Cert in Clinical Pedagogy/MSc Clinical

Pedagogy: https://www.uhi.ac.uk/en/courses/ med-tertiary-and-higher-education/

\section{King's College Hospital NHS}

Foundation Trust and Guy's and St

Thomas' NHS Foundation Trust

RCS Diploma in Orthodontic Therapy

http://dtec.kch.nhs.uk/course/diploma-in-

orthodontic-therapy/

\section{King's College London, University of London}

BDS Dentistry

https://www.kcl.ac.uk/study/undergraduate/ courses/dentistry-bds

BDS Dentistry Graduate/Professional Entry

Programme

https://www.kcl.ac.uk/study/undergraduate/ courses/dentistry-graduate-professionalentry-bds

BSc Dental Therapy \& Hygiene

https://www.kcl.ac.uk/study/undergraduate/ courses/dental-therapy-and-hygiene-bsc MSc Dental Public Health

https://www.kcl.ac.uk/study/postgraduate/ taught-courses/dental-public-health-msc

\section{University of Leeds}

BChD/MChD Dental Surgery/BSc Oral

Science

https://courses.leeds.ac.uk/g033/dentalsurgery-mchd-bchd-oral-science-bsc-mchdbchd-bsc

BSc Dental Hygiene and Dental Therapy https://courses.leeds.ac.uk/i125/dentalhygiene-and-dental-therapy-bsc

\section{University of Liverpool}

BDS Dental Surgery

https://www.liverpool.ac.uk/study/

undergraduate/courses/dental-surgery-bds/

entry-requirements/

Year 0 Foundation to Health and Veterinary Studies

https://www.liverpool.ac.uk/study/

undergraduate/courses/foundation-to-healthstudies-year-0/overview/

$\mathrm{BSc}$ (Hons) Dental Therapy

https://www.liverpool.ac.uk/study/

undergraduate/courses/dental-hygiene-and-

dental-therapy-bsc/overview/

\section{University of Manchester}

BDS Dentistry (first-year entry) https://www.manchester.ac.uk/study/ undergraduate/courses/2021/00398/bdsdentistry-first-year-entry/ BDS Dentistry (pre-dental year entry) https://www.manchester.ac.uk/study/ undergraduate/courses/2021/00399/bdsdentistry-pre-dental-entry/

BSc Oral Health Science

https://www.manchester.ac.uk/study/ undergraduate/courses/2021/00626/bsc-oralhealth-science/

\section{Manchester University NHS}

Foundation Trust - The School for Dental Care Professionals

Diploma in Orthodontic Therapy https://mft.nhs.uk/dental/careers/schoolfor-dental-care-professionals/diploma-inorthodontic-therapy-dip-ortho-ther-rcsed/

\section{Newcastle University}

BDS Dental Surgery

https://www.ncl.ac.uk/undergraduate/2021/ degrees/a206/

$\mathrm{BSc}$ (Hons) Oral and Dental Health Sciences (Dental Hygiene and Therapy) https://www.ncl.ac.uk/undergraduate/ degrees/a207/

\section{New College Lanarkshire}

BSc Dental Nursing

https://www.nclanarkshire.ac.uk/courses/ dental-nursing/bsc-dental-nursing

\section{Nottingham College}

FdSc (Foundation Degree Level 5) Dental Technology

https://www.nottinghamcollege.ac.uk/ courses/dental-technology-foundationdegree-level-5-full-time

\section{University of Plymouth}

BDS Dental Surgery

https://www.plymouth.ac.uk/courses/ undergraduate/bds-dental-surgery BSc(Hons) Dental Therapy and Hygiene https://www.plymouth.ac.uk/courses/ undergraduate/bsc-dental-therapy-hygiene $\mathrm{BSc}$ (Hons) Dental Therapy and Hygiene with Integrated Foundation Year https://www.plymouth.ac.uk/courses/ undergraduate/bsc-dental-therapy-andhygiene-with-integrated-foundation-year

\section{University of Portsmouth}

BSc Dental Hygiene

https://www.port.ac.uk/study/courses/bschons-dental-hygiene
BSc(Hons) Dental Hygiene and Dental Therapy

https://www.port.ac.uk/study/courses/bschons-dental-hygiene-and-dental-therapy CertHE Dental Nursing

https://www.port.ac.uk/study/courses/certhedental-nursing

Dental Development Programme

https://www.port.ac.uk/study/courses/dentaldevelopment-programme

\section{Queen's University Belfast}

BDS Dentistry

https://www.qub.ac.uk/courses/

undergraduate/dentistry-bds-a200/

\section{University of Sheffield}

BDS Dental Surgery

https://www.sheffield.ac.uk/prospectus/

courseDetails.do?id=A2002021

Higher Education Diploma Dental Hygiene and Dental Therapy

https://www.sheffield.ac.uk/dentalschool/ modules/higher-education-diploma-dentalhygiene-and-dental-therapy

BSc Bio-dental Science and Technology https://www.sheffield.ac.uk/prospectus/ courseDetails.do?id=B7502021

\section{Teeside University}

CertHE Dental Nurse Practice https://www.tees.ac.uk/undergraduate_ courses/Nursing_Midwifery_\&_Health_ Professions/Cert_HE_Dental_Nurse_ Practice.cfm

BSc Dental Hygiene and Dental Therapy More information via www.tees.ac.uk Foundation Year (Health) https://www.tees.ac.uk/undergraduate_ courses/Nursing_Midwifery_\&_Health_ Professions/Foundation_Year_(Health).cfm

\section{Further information: Orthodontic therapy}

https://www.gdc-uk.org/education-cpd/ quality-assurance/recent-inspections/ orthodontic-therapy

https://www.rcseng.ac.uk/educationand-exams/exams/search/diploma-inorthodontic-therapy/

Further postgraduate courses in specific areas of dentistry are also available but have generally not been included in this list. 